Check for updates

Cite this: Chem. Sci., 2019, 10, 179

๑ All publication charges for this article have been paid for by the Royal Society of Chemistry

Received 8th July 2018

Accepted 1st October 2018

DOI: $10.1039 / \mathrm{c} 8 \mathrm{sc} 03019 \mathrm{~g}$

rsc.li/chemical-science

\title{
The methylation effect in prolonging the pure organic room temperature phosphorescence lifetime $\uparrow$
}

\author{
Zhu Mao, $t^{\mathrm{a}}$ Zhan Yang, $t^{\mathrm{a}}$ Zhenguo Fan, ${ }^{\mathrm{a}}$ Eethamukkala Ubba, ${ }^{a}$ Wenlang Li, ${ }^{a}$ Yang Li, \\ Juan Zhao, ${ }^{\text {a }}$ Zhiyong Yang, ${ }^{a}$ Matthew P. Aldred ${ }^{a}$ and Zhenguo Chi $\mathbb{D}^{\text {a }}$
}

\begin{abstract}
Prolonging the phosphorescence lifetime of pure organic phosphorescent materials by a methyl-substitution strategy is described. We present a chemical strategy for improving the phosphorescence lifetime of triplet excitons under ambient conditions by incorporating methyl groups into the chemical structures. This is observed by a long-lived phosphorescence lifetime of up to $0.83 \mathrm{~s}$ detected for methylated 9-(4(mesitylsulfonyl)phenyl)-9H-carbazole (3M), compared to $0.36 \mathrm{~s}$ for 9-(4-(phenylsulfonyl)phenyl)-9Hcarbazole (OM) without any methyl groups. Additionally, enhanced phosphorescence efficiency can be obtained at an appropriate methylation degree, because of the smaller $\Delta E_{\mathrm{ST}}$ (singlet and triplet energy gap) and $\Delta E_{T^{*} *}$ (normal phosphorescence and long-lived phosphorescence energy gap). A comprehensive investigation on the packing mode in the crystalline state reveals that the methyl groups occupy the free volume and result in a suppression of non-radiative decay, accounting for the enhanced phosphorescence lifetime.
\end{abstract}

\section{Introduction}

Materials with long-lived phosphorescence emission exhibit extraordinarily long lifetimes, typically a lifetime of $0.1 \mathrm{~s}$ or greater, ${ }^{1}$ and have a wide range of applications, such as in bio-imaging, ${ }^{2}$ sensors, ${ }^{3}$ anti-counterfeiting, ${ }^{4}$ and displays. ${ }^{5}$ However, most of these phosphorescent luminogens are limited to inorganic materials with resource-limited transition metals, such as metal sulphides and alkaline earth aluminates/silicates. ${ }^{6}$ In addition, the preparation processes associate with consuming energy for high temperature sintering and products have high bio-toxicity. Considering these drawbacks of inorganic persistent luminescent materials, one alternative is the utilization of organic long-lived phosphorescence materials. Currently, strategies such as guest-host doping, ${ }^{7}$ constructing organic frameworks ${ }^{8}$ and H-type aggregation ${ }^{9}$ have been proposed to provide a rigid environment for suppressing nonradiative decay of triplet excitons and thus extending the lifetime of organic long-lived phosphorescence. For a higher

${ }^{a}$ PCFM Lab, GDHPPC Lab, Guangdong Engineering Technology, Research Center for High-performance Organic and Polymer Photo-electric, Functional Films, State Key Laboratory of OEMT, School of Chemistry, Sun Yat-Sen University, Guangzhou 510275, China

${ }^{b}$ Instrumental Analysis and Research Center, Sun Yat-Sen University, Guangzhou 510275, China.E-mail: zhaoj95@mail.sysu.edu.cn

$\dagger$ Electronic supplementary information (ESI) available. CCDC 1833075 and 1833083. For ESI and crystallographic data in CIF or other electronic format see DOI: $10.1039 / \mathrm{c} 8 \mathrm{sc} 03019 \mathrm{~g}$

\$ Dr Z. Mao and Dr Z. Yang contributed equally to this work. quantum yield $\left(\Phi_{\mathrm{P}}\right)$ of organic phosphorescence, fast-radiative decay $\left(k_{\mathrm{r}}^{T}\right)$, lower non-radiative decay $\left(k_{\mathrm{nr}}{ }^{T}\right)$ and higher intersystem crossing (ISC) efficiency ( $\Phi_{\text {ISC }}$ ) are the key parameters $\left(\Phi_{\mathrm{P}}=k_{\mathrm{r}}^{T} /\left(k_{\mathrm{r}}^{T}+k_{\mathrm{nr}}{ }^{T}\right) \Phi_{\mathrm{ISC}}\right) \cdot{ }^{10}$ However, there is a contradiction in that fast-radiative decay means a short phosphorescence lifetime $\left(\tau_{\mathrm{p}}\right)$. Therefore, the balance between phosphorescence lifetime and $\Phi_{\mathrm{P}}$ is pivotal to the development of high performance organic long-lived phosphorescence emitters, and some strategies including heavy atom effects, ${ }^{11}$ dihydrogen bonding, ${ }^{12}$ and polycrystalline phases ${ }^{13}$ have been previously reported. In spite of this progress, further detailed studies regarding the prolonging of the phosphorescence lifetime and enhancing the phosphorescence quantum efficiency are still required.

Methylation is an important process in bio-systems and disrupted DNA methylation affecting the genes in cells is associated with multiple diseases, ${ }^{\mathbf{1 4}}$ indicating a shorter life. The methyl group is a weak donor and relatively bulky; therefore, we proposed that the methylation of organic semiconductors could have a significant impact on the exciton behaviours of organic phosphorescence materials, such as lifetime and quantum yield efficiency. To validate this hypothesis, herein we presented an investigation into the effect of methyl groups on the phosphorescence lifetime and efficiency of long-lived phosphorescence materials. Cz-DPS (0M), which emits long-lived phosphorescence with a lifetime $\tau_{\mathrm{p}}=0.36 \mathrm{~s}$ under ambient conditions, was reported by our group in 2016. ${ }^{11 b}$ Here, we have observed significant changes in the optical and photo-physical properties when the chemical structure of $\mathbf{0 M}$ is altered by methylation (Fig. 1). Two 


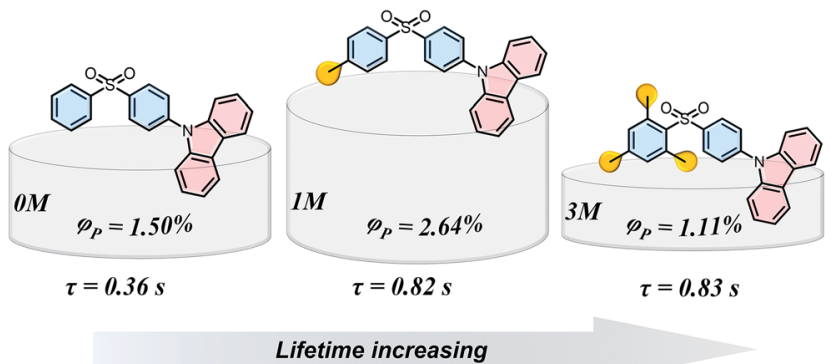

Fig. 1 Chemical structures, long-lived phosphorescence lifetime $(\tau)$, and phosphorescence efficiency $\left(\Phi_{\mathrm{P}}\right)$ of $0 \mathrm{M}, 1 \mathrm{M}$, and $3 \mathrm{M}$.

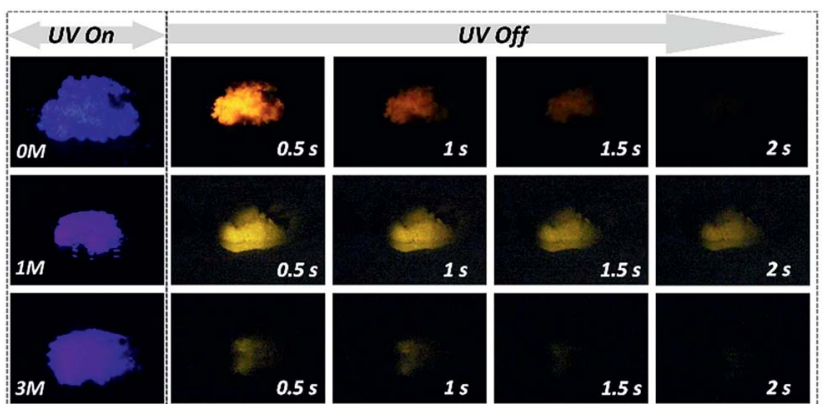

Fig. 2 The long-lived phosphorescence photograph of OM (upper), $1 \mathrm{M}$ (middle), and 3M (bottom) with different delay times.

compounds $\mathbf{1 M}$ and $\mathbf{3} \mathbf{M}$, containing one and three methyl groups, respectively, exhibit long-lived phosphorescence lifetime with $120 \%\left(\tau_{\mathrm{p}}=0.82 \mathrm{~s}\right)$ and $130 \%\left(\tau_{\mathrm{p}}=0.83 \mathrm{~s}\right)$ enhancement compared to $\mathbf{0 M}$ (Fig. 1 and 2). Moreover, the $\Phi_{\mathrm{P}}$ of $\mathbf{1 M}$ shows a significant improvement $(2.64 \%)$ compared to unsubstituted $\mathbf{0 M}(1.50 \%)$.

\section{Results and discussion}

Compounds $\mathbf{1 M}$ and $\mathbf{3} \mathbf{M}$ were both synthesized as shown in Fig. $\mathrm{S} 1 \dagger$ in two-step chemical transformations starting from commercially available sulfonyl chlorides. To explore the changes in the photophysical properties of the derived compounds, we performed systematic investigations in both the solution- and the crystalline-state. In dilute THF solution (1 $\times 10^{-5} \mathrm{M}$ ), as the methylation degree increases the maximum emission peak is slightly blue-shifted (Fig. 3a). This is attributed to the electron-donating ability of the methyl group(s). The fluorescence lifetimes in THF solutions for $\mathbf{0 M}, \mathbf{1 M}$ and $\mathbf{3 M}$ are $6.84 \mathrm{~ns}, 6.83 \mathrm{~ns}$ and $6.39 \mathrm{~ns}$, respectively (Fig. 3b). Furthermore, the theoretical simulation for the energy level distribution, carried out in a cavity of a tetrahydrofuran (THF) solvent and using the polarizable continuum model, for $\mathbf{0 M}, \mathbf{1} \mathbf{M}$ and $\mathbf{3} \mathbf{M}$, is as shown in Fig. S2. $\dagger$ These results suggest that the charge distribution is only slightly affected by the number of methyl groups for the isolated molecules.

Crystalline powders of the three compounds were obtained by recrystallization from a dichloromethane and ethanol solvent mixture (confirmed by powder X-ray diffraction,
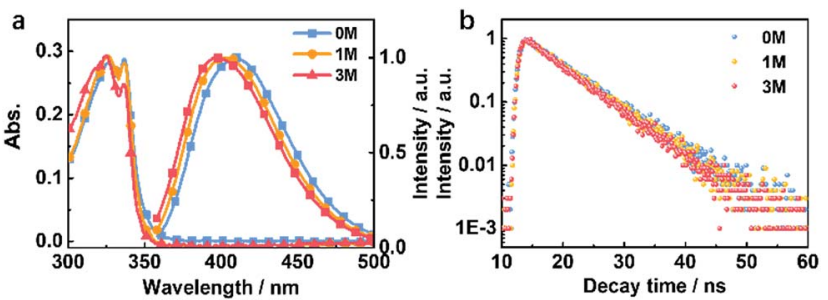

Fig. 3 (a) Absorption and fluorescence (300 K) spectra and (b) fluorescence lifetime decay $(300 \mathrm{~K})$ curves of $0 \mathrm{M}, 1 \mathrm{M}$, and $3 \mathrm{M}$ in $\mathrm{THF}$ solution $\left(10^{-5} \mathrm{M}\right)$.

Fig. $\mathrm{S} 3 \uparrow)$ and display notable differences in optical properties, especially with regard to $\mathbf{1 M}$ and $\mathbf{3 M}$. The maximum emission peaks of $\mathbf{1 M}$ in the crystalline-state and solution-state are at $423 \mathrm{~nm}$ (Fig. 4) and $402 \mathrm{~nm}$, respectively, with a bathochromic shift of $21 \mathrm{~nm}$ for the crystalline-state which can be ascribed to $\pi-\pi$ stacking. ${ }^{9 d}$ For compound $\mathbf{3} \mathbf{M}$, in the crystalline-state the emission peak is located at $376 \mathrm{~nm}$ and in the solution-state it is located at $399 \mathrm{~nm}$, with a hypsochromic shift of $23 \mathrm{~nm}$ of the crystalline-state that is possibly due to $\mathrm{H}$-aggregation. ${ }^{9 a}$ These stacking modes in $\mathbf{1 M}$ and $\mathbf{3} \mathbf{M}$ can be destroyed by grinding and show blue- and red-shifted emission in $\mathbf{1} \mathbf{M}$ and $\mathbf{3} \mathbf{M}$, respectively, as shown in Fig. S4. $\dagger$ Compounds $\mathbf{1} \mathbf{M}$ and $\mathbf{3} \mathbf{M}$ show fluorescence in the crystalline-state with a lifetime of $9.2 \mathrm{~ns}$ and $2.6 \mathrm{~ns}$, respectively (Fig. S5†). The longer fluorescence lifetime of $1 \mathbf{M}$ in the crystalline state might be due to the smaller overlap integral of the frontier orbitals of the monomer (Fig. S6 $\dagger$ ) and $\pi-\pi$ stacking of the dimer (Fig. S7 $\dagger$ ). ${ }^{9 d}$ Remarkably, for the three materials under ambient conditions, yellowish long-lived phosphorescence emission can be easily observed by the naked eye when the UV-light excitation source is removed (Fig. 2). For 1M, delayed phosphorescence is observed and the maximum emission peak is located in a longer wavelength region $(546 \mathrm{~nm})$ with a lifetime $(\tau)$ of $0.82 \mathrm{~s}$, whilst the delayed phosphorescence for $\mathbf{3 M}$ is located at $542 \mathrm{~nm}$ with a lifetime $(\tau)$

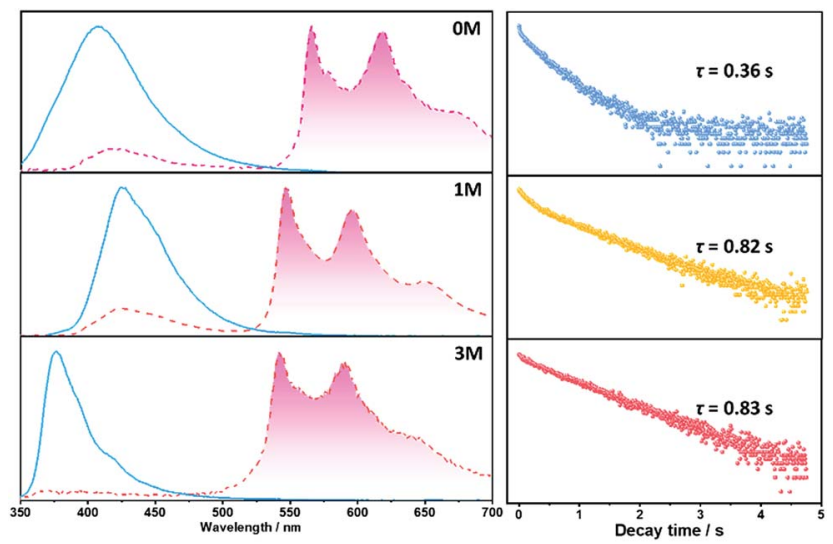

Fig. 4 Steady state fluorescence (blue solid line), long-lived phosphorescence (red dashed line filled with red, delay time $=8 \mathrm{~ms}$ ) spectra of $0 \mathrm{M}, 1 \mathrm{M}$ and $3 \mathrm{M}$, and corresponding maximum emission peak ( $565 \mathrm{~nm}, 546 \mathrm{~nm}$ and $542 \mathrm{~nm}$ for $0 \mathrm{M}, 1 \mathrm{M}$, and $3 \mathrm{M}$, respectively) of long-lived phosphorescence lifetime recorded under ambient conditions with a $350 \mathrm{~nm}$ excitation source. 
of $0.83 \mathrm{~s}$ (Fig. 4, right column). All the long-lived phosphorescence decay behaviours have a mono-exponential shape, which indicates a pure triplet emission centre for the long-lived phosphorescence. With respect to $\mathbf{0 M}$ and $\mathbf{1} \mathbf{M}$, the delayed emission band located in the fluorescence region ( $c a$. 400-450 $\mathrm{nm}$ ) is attributed to the triplet-triplet annihilation (TTA) during the decay time of $\mathbf{0 M}$ and $\mathbf{1} \mathbf{M}$. These results indicate that the methyl groups do have significant influence over the emission intensity and prolong the existence of the long-lived phosphorescence in the crystalline-states.

For further understanding the mechanism of the long-lived phosphorescence of these compounds, the delayed emission was recorded at $77 \mathrm{~K}$ (Fig. 5) in the crystalline-state and the clear changes of the emission bands around $450-530 \mathrm{~nm}$ at room temperature were seen (Fig. 4, red dashed line). We assume that the phosphorescence emission from the isolated molecule is due to normal phosphorescence. With regard to $1 \mathbf{M}$ and $\mathbf{3 M}$, the maximum emission wavelength of the long-lived phosphorescence is $546 \mathrm{~nm}(2.27 \mathrm{eV})$ and $542 \mathrm{~nm}(2.28 \mathrm{eV})$, respectively, and is much more red-shifted than that of their corresponding normal phosphorescence. The longer wavelength phosphorescence suggests the existence of a lower excited state in the crystalline-state with a much longer lifetime compared to the isolated molecules, which is due to the electronic coupling of adjacent molecules (dimer) in the crystals..$^{\mathbf{9}, \mathbf{1 1 b}}$ Single crystals of $\mathbf{1 M}$ and $\mathbf{3 M}$ were obtained by slow evaporation of saturated solutions from mixed solvents (dichloromethane and ethanol) under the same conditions for the crystalline powders. Single crystal X-ray diffraction was carried out to gain information regarding the packing of the methylated molecules (Fig. S7 and $\mathrm{S} 8 \dagger$ ). The intermolecular interactions between adjacent molecular units in the acquired crystals containing $\mathrm{C}-\mathrm{H} \cdots \pi$,

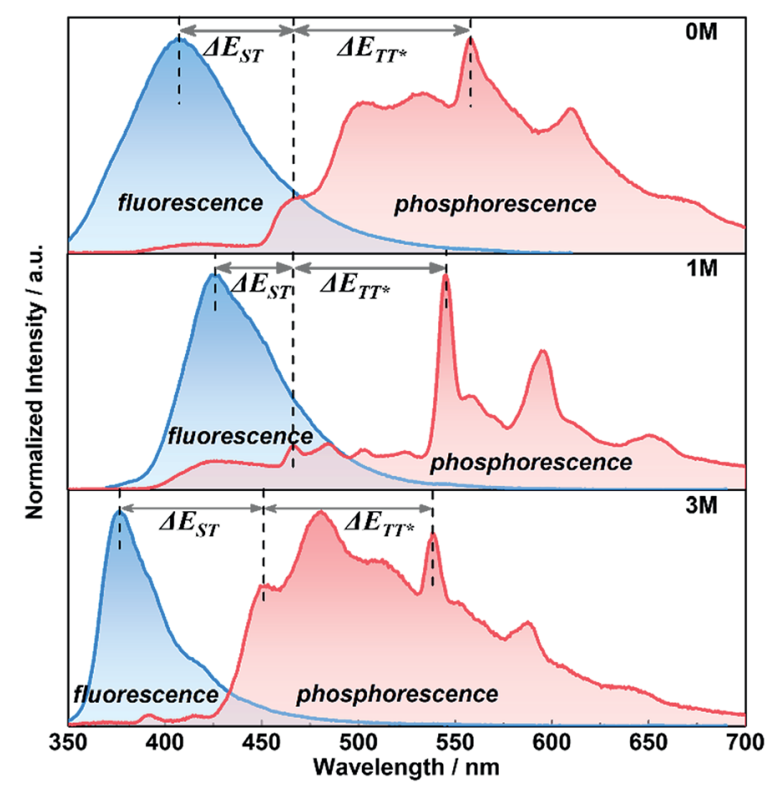

Fig. 5 Fluorescence (blue area, $300 \mathrm{~K}$ ) and phosphorescence spectra (red area, $77 \mathrm{~K}$, and delay time $=8 \mathrm{~ms}$ ) for $0 \mathrm{M}, 1 \mathrm{M}$, and $3 \mathrm{M}$ from up to bottom. $\Delta E_{\mathrm{ST}}=$ energy gap between the participating $\mathrm{S}_{1}$ and $\mathrm{T}_{1}$ states and $\Delta E_{T^{*}}=$ stabilized gap.
$\mathrm{S}=\mathrm{O} \cdots \mathrm{H}$ and $\pi-\pi$ stacking cause the construction of the crystalline frameworks. These intermolecular interactions can help suppress the non-radiative pathways of excited molecules. As a consequence, the three crystals emit bright blue fluorescence with relatively high fluorescence quantum yields of $29.1 \%$, $72.5 \%$ and $51.1 \%$ for $\mathbf{0 M}, \mathbf{1 M}$, and $\mathbf{3 M}$, respectively. Compound $\mathbf{1 M}$ shows close contact between neighbouring molecules, i.e., co-linear by the head-to-tail style with $\mathrm{C}-\mathrm{H} \cdots \pi(2.719 \AA)$ forming dimer 1, taking the dipole orientation into account, as H-type interaction. The molecular arrangements of the face-to-face interactions reveal that the $\pi-\pi$ stacking (3.464 $\AA$ ) mode aids the construction of dimer 2 and the two adjacent units adopting the anti-parallel packing mode with $\mathrm{S}=\mathrm{O} \cdots \mathrm{H}$ interaction (2.633 A) forming dimer 3. These numerous intermolecular interactions contribute to the comparatively high fluorescence quantum yield of $\mathbf{1 M}$ in the crystalline state. Likewise, the $\mathrm{S}=\mathrm{O} \cdots \mathrm{H}$ interactions constructed the framework of the $\mathbf{3 M}$ single crystals consisting of the three kinds of dimers (Fig. S8 $\dagger$ ). Molecules with such plenty of interactions in the crystal lattices improve the potential for phosphorescence emission.

Theoretical time-dependent density functional theory (TDDFT) calculation studies have been carried out to reveal the intermolecular electronic coupling effects in the dimer molecule system. ${ }^{15}$ As discussed in our previous work, ${ }^{11 b}$ the different electronic configurations of the donor (D) and acceptor (A) units make a significant contribution to enhancing the intermolecular electronic coupling (IEC) effects. Hence, we extracted the dimers containing spatially close donor and acceptor units (both dimer $\mathbf{1}$ for $\mathbf{1 M}$ and $\mathbf{3 M}$, Fig. $\mathbf{S} 8 \dagger$ ) to evaluate the IEC effects and identify the possible ISC channels in both monomers and dimers (Fig. S9†). The monomers of $\mathbf{0 M}, \mathbf{1 M}$, and $\mathbf{3 M}$ exhibit an increased energy gap between the lowest excited singlet $\left(\mathrm{S}_{1}\right)$ and triplet $\left(\mathrm{T}_{1}\right)$ states, demonstrating that the ability of electron-withdrawing nature of the diphenylsulphone group is reduced by methylation. For instance, the $T_{1}, T_{4}, T_{7}$, and $T_{9}$ states of the isolated $\mathbf{1} \mathbf{M}$ molecules involve the same orbital composition as the $\mathrm{S}_{1}$ state, indicating good channels for ISC. ${ }^{\mathbf{9}, \mathbf{1 1 b} \boldsymbol{1 5 a} \boldsymbol{a}}$ Considering the narrower energy gap between the $\mathrm{S}_{\mathbf{1}}$ and $\mathrm{T}_{n}$ states, the ISC channels become more efficient in the dimer models. The energy levels of $T_{7}$ and $S_{1}$ states are relatively close in the $\mathbf{1 M}$ dimer, and the orbital composition is also well matched, indicating a barrier-less intersystem crossing pathway between $S_{1}$ and $T_{7}$. The hot excitons $\left(T_{7}\right)$ decay to the lowest triplet state via rapid internal conversion (IC) and then to the ground state, resulting in long-lived phosphorescence at room temperature in $\mathbf{1 M}$ with $\Phi_{\mathrm{P}}=2.64 \%$. Compared to the $\mathbf{1} \mathbf{M}$ dimer, compound $\mathbf{3} \mathbf{M}$ shows fewer and lower efficiency ISC channels (Fig. S9†), resulting in a relatively low ISC efficiency and contributing to the lowest long-lived phosphorescence efficiency $\left(\Phi_{\mathrm{P}}=1.11 \%\right)$ amongst the methylated systems.

By combining the photophysical properties and the packing modes as well as the ISC channels in the single crystals, we have proposed a mechanism for the long-lived phosphorescence in these methylated derivatives (Fig. 6). The short lifetime fluorescence of several nanoseconds results from the radiative decay of the lowest excited singlet state $\left(\mathrm{S}_{1}\right)$, which can also form triplet excitons through ISC with the help of the electronic 

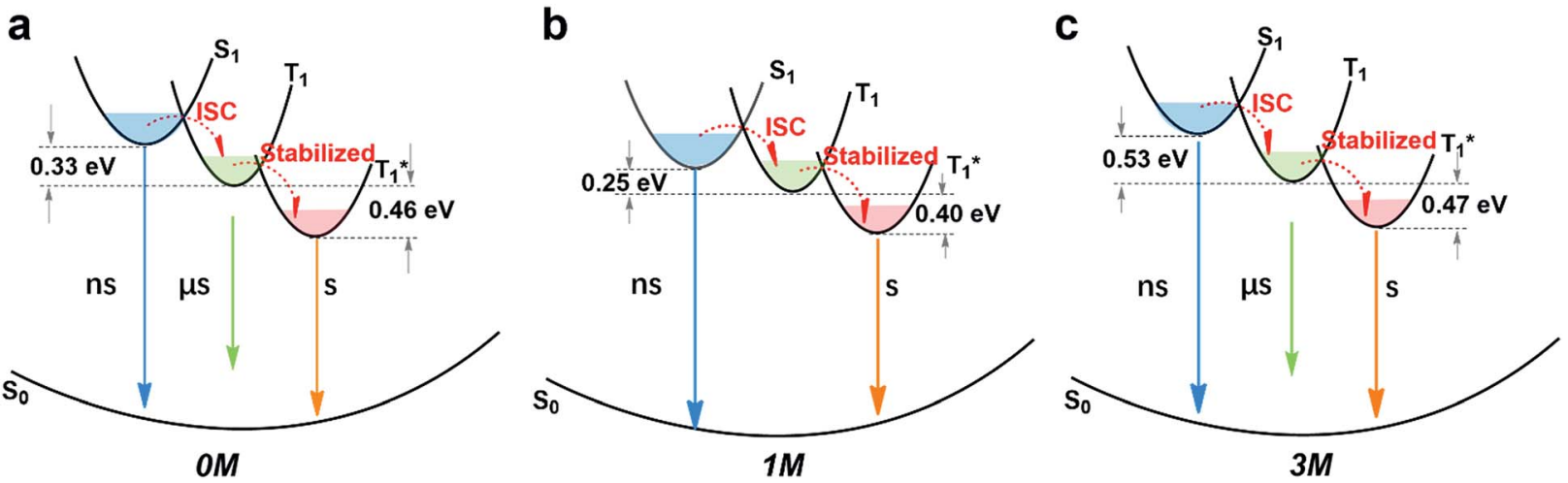

Fig. 6 Proposed mechanism of long-lived phosphorescence and energy split ( $\Delta E_{\mathrm{ST}}$ and $\Delta E_{\mathrm{TT} *}$ ) of (a) $0 \mathrm{M}$, (b) $1 \mathrm{M}$, and (c) 3M. Blue arrows indicate fluorescence, green arrows represent normal phosphorescence, and yellow arrows represent long-lived phosphorescence.

configuration according to El-Sayed's rule, ${ }^{16}$ enabling the occurrence of the normal phosphorescence with typical microseconds to milliseconds lifetime. The long-lived phosphorescence (hundreds of milliseconds) is observed in crystals due to the presence of a new lower-lying triplet energy level $\left(\mathrm{T}_{1}^{*}\right)$, which is formed by the interaction of adjacent molecules and refers to as IEC. ${ }^{11 b}$ In these processes, the splitting of energy between two different excited states shows a great influence on the transition probability. For example, the narrow gap can facilitate the processes of ISC and IC according to the Arrhenius equation. ${ }^{17}$ As calculated from the fluorescence and cryogenic phosphorescence spectra (Fig. 5), the energy splitting of $\Delta E_{\mathrm{ST}}(0.25 \mathrm{eV})$ and $\Delta E_{\mathrm{TT}^{*}}(0.40 \mathrm{eV})$ of $\mathbf{1 M}$ is minimal (Fig. 6), thus facilitating exciton population at the $\mathrm{T}_{1}^{*}$ state in crystals. A higher exciton concentration of $\mathbf{1 M}$ generates brighter long-lived phosphorescence. Compound $3 \mathrm{M}$ exhibits a larger $\Delta E_{\mathrm{ST}}(0.53 \mathrm{eV})$ and $\Delta E_{\mathrm{TT}^{*}}$ $(0.47 \mathrm{eV})$, showing relatively weaker long-lived phosphorescence as depicted in Fig. 2. Interestingly, the emission of normal phosphorescence $\left(T_{1}\right)$ in $\mathbf{0 M}(467 \mathrm{~nm}$, the first phosphorescence peak confirmed at $77 \mathrm{~K}$, Fig. 5) and $3 \mathbf{M}(449 \mathrm{~nm}$, the first phosphorescence peak at $77 \mathrm{~K}$, Fig. 5) under ambient conditions could be detected but is absent in $1 \mathbf{M}$ (Fig. S10 and Table S1 $\dagger$ ), which is due to the smaller $\Delta E_{\mathrm{TT}^{*}}$ in $\mathbf{1 M}$. Additionally, triplet excitons $\left(T_{1}\right)$ generated in $\mathbf{1 M}$ can be stabilized quickly to the lower triplet state $\mathrm{T}_{1}^{*}$ instead of radiative decay, without normal phosphorescence under ambient conditions. The complete transformation of the $\mathrm{T}_{1}$ excitons to the concerned $\mathrm{T}_{1}^{*}$ state enhances the brightness of the long-lived phosphorescence; therefore, $\mathbf{1} \mathbf{M}$ has the highest long-lived phosphorescence quantum efficiency amongst the three reported compounds. Both theoretical calculations and spectra analysis indicate that the methyl group(s) regulate the energy level distribution within the compounds $\mathbf{1 M}$ and $\mathbf{3} \mathbf{M}$, which results in long-lived phosphorescence quantum yield differences.

Besides the quantum efficiency, another crucial parameter for organic room temperature long-lived phosphorescence materials is lifetime, which is the reciprocal of the rate of the natural triplet radiative decay. Lifetime strongly depends on the oscillator strength between the lowest excited triplet and ground states for phosphorescence. An appropriate $\left(\mathrm{n}, \pi^{*}\right)$ configuration proportion can regulate this process and tune the lifetime of pure organic long-lived phosphorescence materials. ${ }^{16}$ In our case, the methyl groups have only slight influence on the electronic configuration for isolated molecules, as proven by their similar spectral profiles, such as absorption, fluorescence and phosphorescence emission. Therefore, this indicates that another factor could be the cause of altering the long-lived phosphorescence lifetimes of the methylated compounds. Moreover, the rigid environment means less structural vibration and is essential for stabilizing the triplet excitons for designing pure organic room temperature phosphorescent materials. ${ }^{18}$ In general, the molecules in a crystal lattice occupy most of the space of a unit cell. However, along with the occupied spaces in crystals, there are plenty of unoccupied spaces, i.e., the free volume, which can afford the structural vibration with nonradiative decay of the excited state. As depicted in Fig. 7, we calculated the free volume distribution of the compounds $\mathbf{0 M}, \mathbf{1} \mathbf{M}$ and $\mathbf{3 M}$ using Materials Studio with a 1.0 Å diameter sensor. Intriguingly, a negative correlation was determined between the long-lived phosphorescence lifetime and fractional free volume $\left(V_{\mathrm{f}}\right)$ of the crystals (Fig. 8 and S9a $\uparrow$ ). As the $V_{\mathrm{f}}$ becomes smaller, longer-lived emission could be observed in the increments of the methylation degree from $\mathbf{0 M}$ to $\mathbf{1 M}$ and to $\mathbf{3} \mathbf{M}$. A diminished $V_{\mathrm{f}}$ in the crystals of $\mathbf{1 M}$ and $\mathbf{3} \mathbf{M}$ indicates a more compact packing pattern, accompanied by less space for the structural relaxation of the excited molecules and thus suppresses the non-radiative decay $\left(k_{\mathrm{nr}}{ }^{T}\right)$. Meanwhile, the long-lived lifetime of these three compounds in the crystalline state shows only a slight increase as the temperature decreases

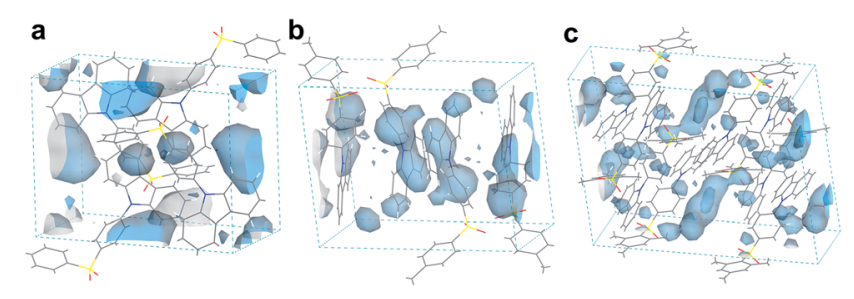

Fig. 7 Free volume region in the single crystal cells of (a) $0 M$, (b) $1 M$, and (c) $3 \mathrm{M}$. 


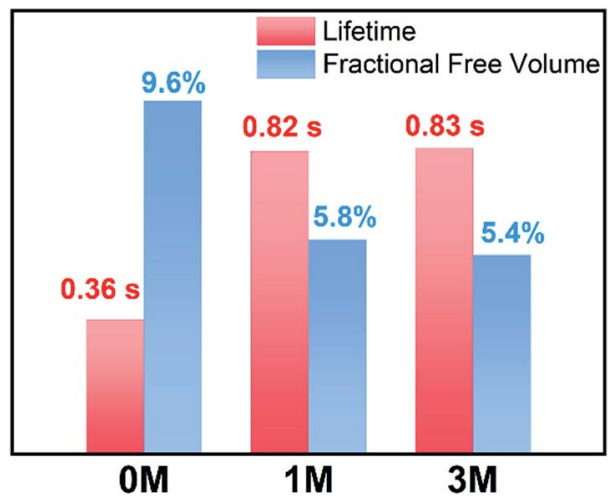

Fig. 8 Fractional free volume and long-lived phosphorescence lifetime of $0 M, 1 M$, and $3 M$.

(Fig. S11 and Table S2 $\dagger$ ), which is in contrast to the normal phosphorescence (continuous decreasing with the temperature increasing). ${ }^{19}$ The near-constant lifetimes at low temperature and room temperature demonstrate the heavy suppression of the non-radiative pathways in these compounds, and the importance of free volume. Consequently, the restricted nonradiative decay leads to long-lived phosphorescence according to the equation $\tau_{\mathrm{p}}=1 /\left(k_{\mathrm{r}}^{T}+k_{\mathrm{nr}}{ }^{T}\right) \cdot{ }^{20}$ On the other hand, the decreasing energy difference between the maximum excitation and the fluorescence emission $\left(\Delta E_{\text {ex-em }}\right)$ of the crystalline solid indicates that there is less energy loss with an increasing number of methyl groups and also the same decreasing trend can be observed in the correlation between $V_{\mathrm{f}}$ and $\Delta E_{\text {ex-em }}$ (Fig. S12b and S13 $\uparrow$ ). Less excited state structural vibrations for $\mathbf{1 M}$ and $\mathbf{3 M}$ are also evident from the narrower fluorescence full width at half maximum (Fig. 4, blue lines) compared to $\mathbf{0 M} .^{21}$

Furthermore, we compared the $V_{\mathrm{f}}$ and lifetime of several reported typically long-lived phosphorescence compounds (Fig. S14 and Table S3 $\dagger$ ). A similar noticeable negative trend between $\tau_{\mathrm{p}}$ and $V_{\mathrm{f}}$ was observed (dashed line in Fig. S14 $\dagger$ ). These results suggest that $V_{\mathrm{f}}$ has a crucial impact on the phosphorescence lifetime. The negative correlation of $V_{\mathrm{f}}$ is very similar to the heavy atom effect in phosphorescent emitters. However, the difference is that the methylation effect accelerates the non-radiative pathways, whilst the heavy atom effect enhances the radiative pathways. ${ }^{10}$ Inspired by the concept of the life-span based on the methylation in bio-systems, the relationship between $V_{\mathrm{f}}$ and $\tau_{\mathrm{p}}$ proves that the incorporation of methyl groups decreases the free volume in the crystal lattice and confines the excited molecular vibrational relaxations, resulting in the enhancement of the longlived phosphorescence lifetimes of purely organic phosphorescent emitters. Finally, we have also synthesized additional acridine-type related compounds (DMAC-0M and DMAC-1M) to demonstrate that the methylation effect can also be expanded to other phosphorescence emitters (Fig. S15 $\dagger$ ). Indeed, for these DMAC-based compounds the phosphorescence lifetime is also enhanced upon methylation, among which DMAC-0M that contains no methyl group exhibits a lifetime of $5.30 \mu$ s and DMAC-1M that contains one methyl group exhibits a lifetime of $24.3 \mu$ s (Fig. S16†).

\section{Conclusions}

In summary, we have presented a method for prolonging the lifetime of purely organic room temperature phosphorescence materials. This comprehensive investigation confirms that the incorporation of the methyl group(s) has a crucial effect on the quantum yield and lifetime. Increasing the number of methyl groups prolongs the lifetime at room temperature up to $0.82 \mathrm{~s}$ (1M) and $0.83 \mathrm{~s}(\mathbf{3 M})$ from $0.36 \mathrm{~s}(\mathbf{0 M})$. The methyl groups effectively decrease the fractional free volumes within the crystal lattice and subsequently suppress the non-radiative decay pathways of the excited structural relaxation. However, excessive methylation of the structures enlarges the energy split $\left(\Delta E_{\mathrm{ST}}\right.$ and $\left.\Delta E_{\mathrm{TT}^{*}}\right)$, resulting in a reduction of the phosphorescence quantum yield. Combining the lifetime and quantum yield, we can conclude that an appropriate methylation degree can afford a good balance to improve the integrated performance of long-lived phosphorescence materials.

\section{Conflicts of interest}

There are no conflicts to declare.

\section{Acknowledgements}

This work was financially supported by the National Natural Science Foundation of China (NSFC: 51733010, 61605253 and 21672267), the Science and Technology Planning Project of Guangdong (2015B090913003), the China Postdoctoral Science Foundation (2017M620395) and the Fundamental Research Funds for the Central Universities.

\section{Notes and references}

1 S. Xu, R. Chen, C. Zheng and W. Huang, Adv. Mater., 2016, 28, 9920.

2 (a) Q. Miao, C. Xie, X. Zhen, Y. Lyu, H. Duan, X. Liu, J. V. Jokerst and K. Pu, Nat. Biotechnol., 2017, 35, 1102; (b) S. M. A. Fateminia, Z. Mao, S. Xu, Z. Yang, Z. Chi and B. Liu, Angew. Chem., Int. Ed., 2017, 56, 12160; (c) S. Cai, H. Shi, J. Li, L. Gu, Y. Ni, Z. Cheng, S. Wang, W. W. Xiong, L. Li, Z. An and W. Huang, Adv. Mater., 2017, 29, 1701244; (d) X. Chen, C. Xu, T. Wang, C. Zhou, J. Du, Z. Wang, H. Xu, T. Xie, G. Bi, J. Jiang, X. Zhang, J. N. Demas, C. O. Trindle, Y. Luo and G. Zhang, Angew. Chem., Int. Ed., 2016, 55, 9872; (e) Q. Miao and K. Pu, Adv. Mater., 2018, 1801778.

3 (a) C. Li, X. Tang, L. Zhang, C. Li, Z. Liu, Z. Bo, Y. Q. Dong, Y.-H. Tian, Y. Dong and B. Z. Tang, Adv. Opt. Mater., 2015, 3, 1184; (b) Y. Katsurada, S. Hirata, K. Totani, T. Watanabe and M. Vacha, Adv. Opt. Mater., 2015, 3, 1726.

4 K. Jiang, L. Zhang, J. Lu, C. Xu, C. Cai and H. Lin, Angew. Chem., Int. Ed., 2016, 55, 7231.

5 R. Kabe, N. Notsuka, K. Yoshida and C. Adachi, Adv. Mater., 2016, 28, 655. 
6 (a) A. Jaina, A. Kumara, S. J. Dhoblea and D. R. Peshwea, Renewable Sustainable Energy Rev., 2016, 65, 135; (b) J. Hölsä, Electrochem. Soc. Interface, 2009, 18, 42.

7 (a) J. Wei, B. Liang, R. Duan, Z. Cheng, C. Li, T. Zhou, Y. Yi and Y. Wang, Angew. Chem., Int. Ed., 2016, 55, 15589; (b) R. Gao and D. Yan, Chem. Sci., 2017, 8, 590; (c) S. Hirata and M. Vacha, J. Phys. Chem. Lett., 2016, 7, 1539; (d) N. Notsuka, R. Kabe, K. Goushi and C. Adachi, Adv. Funct. Mater., 2017, 27, 1703902; (e) R. Kabe and C. Adachi, Nature, 2017, 550, 384.

8 (a) X. G. Yang and D. P. Yan, Adv. Opt. Mater., 2016, 4, 897; (b) H. Mieno, R. Kabe, N. Notsuka, M. D. Allendorf and C. Adachi, Adv. Opt. Mater., 2016, 4, 1015; (c) S. Cai, H. Shi, Z. Zhang, X. Wang, H. Ma, N. Gan, Q. Wu, Z. Cheng, K. Ling, M. Gu, C. Ma, L. Gu, Z. An and W. Huang, Angew. Chem., Int. Ed., 2018, 57, 4005.

9 (a) Z. An, C. Zheng, Y. Tao, R. Chen, H. Shi, T. Chen, Z. Wang, H. Li, R. Deng, X. Liu and W. Huang, Nat. Mater., 2015, 14, 685; (b) J. A. Li, J. Zhou, Z. Mao, Z. Xie, Z. Yang, B. Xu, C. Liu, X. Chen, D. Ren, H. Pan, G. Shi, Y. Zhang and Z. Chi, Angew. Chem., Int. Ed., 2018, 57, 6449; (c) X. Zhen, Y. Tao, Z. An, P. Chen, C. Xu, R. Chen, W. Huang and K. Pu, Adv. Mater., 2017, 29, 1606665; (d) S. Pan, Z. Chen, X. Zheng, D. Wu, G. Chen, J. Xu, H. Feng and Z. Qian, J. Phys. Chem. Lett., 2018, 9, 3939.

10 A. Köhler and H. Bässler, Electronic Processes in Organic Semiconductors, An Introduction, 2015.

11 (a) S. Cai, H. Shi, D. Tian, H. Ma, Z. Cheng, Q. Wu, M. Gu, L. Huang, Z. An, Q. Peng and W. Huang, Adv. Funct. Mater., 2018, 28, 1705045; (b) Z. Yang, Z. Mao, X. Zhang, D. Ou, Y. Mu, Y. Zhang, C. Zhao, S. Liu, Z. Chi, J. Xu,
Y. C. Wu, P. Y. Lu, A. Lien and M. R. Bryce, Angew. Chem., Int. Ed., 2016, 55, 2181; (c) P. Xue, P. Wang, P. Chen, B. Yao, P. Gong, J. Sun, Z. Zhang and R. Lu, Chem. Sci., 2017, 8, 6060.

12 L. Gu, H. Shi, C. Miao, Q. Wu, Z. Cheng, S. Cai, M. Gu, C. Ma, W. Yao, Y. Gao, Z. An and W. Huang, J. Mater. Chem. C, 2018, 6, 226.

13 Y. Xie, Y. Ge, Q. Peng, C. Li, Q. Li and Z. Li, Adv. Mater., 2017, 29, 1606829.

14 B. P. Williams and M. Gehring, Nat. Commun., 2017, 8, 2124. 15 (a) L. Paul, S. Chakrabarti and K. Ruud, J. Phys. Chem. Lett., 2017, 8, 1253; (b) H. Ma, W. Shi, J. Ren, W. Li, Q. Peng and Z. Shuai, J. Phys. Chem. Lett., 2016, 7, 2893.

16 W. Zhao, Z. He, J. W. Y. Lam, Q. Peng, H. Ma, Z. Shuai, G. Bai, J. Hao and B. Z. Tang, Chem, 2016, 1, 592.

17 (a) X. Han, Q. Bai, L. Yao, H. Liu, Y. Gao, J. Li, L. Liu, Y. Liu, X. Li, P. Lu and B. Yang, Adv. Funct. Mater., 2015, 25, 7521; (b) Q. Zhang, B. Li, S. Huang, H. Nomura, H. Tanaka and C. Adachi, Nat. Photonics, 2014, 8, 326.

18 (a) R. Gao, X. Fang and D. Yan, J. Mater. Chem. C, 2018, 6, 4444; (b) P. Xue, J. Sun, P. Chen, P. Wang, B. Yao, P. Gong, Z. Zhang and R. Lu, Chem. Commun., 2015, 51, 10381.

19 B. Xu, H. Wu, J. Chen, Z. Yang, Z. Yang, Y. C. Wu, Y. Zhang, C. Jin, P. Y. Lu, Z. Chi, S. Liu, J. Xu and M. Aldred, Chem. Sci., 2017, 8, 1909.

20 J. Yang, X. Zhen, B. Wang, X. Gao, Z. Ren, J. Wang, Y. Xie, J. Li, Q. Peng, K. Pu and Z. Li, Nat. Commun., 2018, 9, 840.

21 J. S. Ward, R. S. Nobuyasu, A. S. Batsanov, P. Data, A. P. Monkman, F. B. Dias and M. R. Bryce, Chem. Commun., 2016, 52, 2612. 